\title{
ENTOMOFAUNA DEL PARQUE NACIONAL LOMA NALGA DE MACO Y ALREDEDORES, PROVINCIA ELÍAS PIÑA, REPÚBLICA DOMINICANA
}

\author{
Daniel E. Perez-Gelabert ${ }^{1}$, Ruth H. Bastardo ${ }^{2}$ y Sardis Medrano ${ }^{3}$ \\ ${ }^{1}$ Department of Entomology, U. S. National Museum of Natural History, \\ Smithsonian Institution, P. O. Box 37012, Washington, DC 20013-7012, USA.perezd@si.edu; \\ ${ }^{2}$ Instituto de Investigaciones Botánicas y Zoológicas, Universidad Autónoma de Santo Domingo, \\ Santo Domingo, República Dominicana.r bastardo@hotmail.com; \\ ${ }^{3}$ Instituto Dominicano de Investigaciones Agropecuarias y Forestales, Santo Domingo, \\ República Dominicana. medrano_sardis@hotmail.com
}

\section{RESUMEN}

Se compilaron datos derivados principalmente de la literatura zoológica publicada sobre la diversidad de insectos conocida del Parque Nacional Loma Nalga de Maco y alrededores, en el noroeste de la República Dominicana. Se encontraron 133 especies pertenecientes a 9 órdenes de insectos. Se recomienda la realización de un inventario básico dedicado específicamente a los insectos de esta área protegida.

Palabras clave: insectos, diversidad biológica, inventario de especies, Loma Nalga de Maco.

\section{ABSTRACT}

Data derived mainly from the published zoological literature are compiled on the diversity of insects known from Parque Nacional Loma Nalga de Maco and surroundings located in northwestern Dominican Republic. A total of 133 species belonging to 9 insect orders were found. We recommend a basic inventory especifically dedicated to the insects of this protected area.

Key words: insects, biological diversity, species inventory, Loma Nalga de Maco.

\section{INTRODUCCIÓN}

La importancia de los artrópodos en el equilibrio natural de los ecosistemas es comúnmente poco apreciada. Los artrópodos son el componente más diverso y abundante en la mayoría de ecosistemas terrestres y son fundamentales para la sostenibilidad de los mismos, siendo fuente de alimento para muchas especies, y/o teniendo interacciones vitales (polinización, depredación y parasitismo) con otras. Amenudo, las necesidades más apremiantes en la evaluación y conservación de los artrópodos se presentan en áreas tropicales, donde se encuentran las diversidades más altas y también las amenazas más fuertes. Un manejo orientado a la conservación de un ecosistema está sujeto a la aplicación práctica de conocimientos detallados sobre la biología y dinámica de las especies y sistemas involucrados.

No es sorprendente que gran parte de la fauna de insectos de La Hispaniola sea mal conocida y que existan datos biológicos sobre muy pocas especies. La República Dominicana no tiene tradición de estudios faunísticos. La gran mayoría de los conocimientos que tenemos sobre esta fauna han sido producidos por investigadores extranjeros. Como muchos otros países, la República Dominicana está experimentando grandes cambios en sus ambientes naturales, provocados por perturbaciones, tales como la expansión de las comunidades urbanas, la agricultura intensiva y la introducción de especies exóticas. Estos y otros factores afectan las poblaciones de las 
especies y, a menudo, provocan la extinción local o regional de las más susceptibles. Por esto, la realización de un inventario nacional de especies debería ser una tarea contra el reloj. Un mejor entendimiento de las comunidades naturales en la isla sería particularmente importante en el manejo de las especies, el control de aquellas que son plagas e introducidas y en los estudios de biogeografía.

Como es de esperar, la fauna más conocida y normalmente considerada en los planes de manejo del Parque Nacional Loma Nalga de Maco son los vertebrados, principalmente las aves, reptiles y anfibios. En comparación con la diversidad total estimada en el área, los datos hasta ahora acumulados sobre los insectos y otros artrópodos son pocos y se encuentran completamente dispersos. Este trabajo tiene como objetivo producir una lista de las especies de insectos identificadas de esta área protegida y sus alrededores. Los mismos son sólo el inicio de una caracterización faunística más detallada. Estos censos faunísticos deberán ayudar a identificar a los artrópodos como elementos importantes de las comunidades naturales en la isla.

\section{OBJETIVO}

- Compilar y analizar la información publicada sobre la diversidad de insectos en el Parque Nacional Loma Nalga de Maco, en el noroeste de la República Dominicana.

\section{MATERIALES Y MÉTODOS}

Área de estudio. El Parque Nacional Loma Nalga de Maco, con un área de unos $278 \mathrm{~km}^{2} \mathrm{y}$ situado entre 600 - 1,990 metros sobre el nivel del mar (msnm), se localiza en el extremo noroccidental de la Cordillera Central, en la provincia de Elías Piña, República Dominicana. La vegetación del área incluye bosque latifoliado, bosque nublado y un bosque enano. La cima de la montaña comúnmente se encuentra cubierta de nubes, demostrando su importancia como punto de origen de varios ríos. El municipio de Río Limpio es la comunidad aledaña más importante y es adyacente a su porción noroeste.

Análisis de la literatura. Para detectar las especies de insectos reportadas de este sitio protegido, se examinó la literatura zoológica internacional, principalmente mediante búsquedas electrónicas en Zoological Record. La tarea de identificar trabajos conteniendo esta información también fue grandemente facilitada por el listado general de los artrópodos reportados para La Hispaniola (Perez-Gelabert, 2008).

\section{RESULTADOS Y DISCUSIÓN}

Insectos reportados. El examen de la literatura que contiene reportes de insectos del Parque Nacional Loma Nalga de Maco y alrededores, junto al estudio de algunas colecciones, produjo una lista de 133 especies de insectos (Tabla 1), pertenecientes a nueve (9) órdenes. Los lepidópteros resultaron tener la mayor cantidad de especies (48), seguidos por los tricópteros (27) y los hemípteros (18). La mayoría son especies de distribución relativamente amplia en la isla. En la lista se identifican las especies endémicas de la isla (57) y también se incluye la localidad para la cual se ha registrado la especie en el área. Puede observarse que la lista se compone principalmente de especies colectadas en la zona de Río Limpio y sus alrededores.

Muchas de las mariposas reportadas por Schwartz (1989) son de localidades que distan 12 y 16 kilómetros de Río Limpio. Sólo cinco de las especies en la lista son hasta ahora conocidas exclusivamente de la Loma Nalga de Maco. Tres son luciérnagas del género Heterophotinus 
(Coleoptera: Lampyridae), que probablemente tienen una distribución mucho más amplia. Otra es un saltamontes del género Antillacris (Orthoptera: Episactidae) que habita el bosque nublado en alturas alrededor de los 1,200 msnm. La quinta especie es una mariposa del género Calisto (Lepidoptera: Nymphalidae) sólo colectada una vez en la cima (1,990 msnm) de esta montaña.

Muestreos entomológicos. En términos generales, la fauna de artrópodos en el Parque Nacional Loma Nalga de Maco ha sido poco estudiada. Las pocas colectas entomológicas realizadas han sido esfuerzos aislados por parte de unos pocos colectores. A nuestro saber, los primeros entomólogos extranjeros en visitar Río Limpio fueron Donald y Mignon Davis (U.S. National Museum of Natural History, NMNH, Smithsonian Institution, Washington, DC) en 1973, quienes cuentan que el área era entonces todo un esplendoroso bosque. Además de las mariposas nocturnas en las que se especializan estos autores, colectaron muchos grupos de insectos cuyos especímenes hoy se encuentran repartidos por toda la colección del museo. Pero quizás el investigador que ha realizado las contribuciones más importantes en esta área fue el norteamericano David $\mathrm{K}$. Wetherbee, quien vivió en Montecristi y Dajabón desde principios de la década de 1980 hasta su muerte en 1997. Entre estos años, Wetherbee produjo múltiples trabajos sobre la historia de las exploraciones biológicas en el Caribe y contribuyó significativamente a la taxonomía de las mariposas diurnas de La Hispaniola. Wetherbee parece haber sido quien más tiempo ha dedicado a la exploración faunística de la Loma Nalga de Maco y alrededores. Algunos de sus escritos contienen los datos quizás más completos que se tienen sobre esta área. Uno de sus libros mimeografiados (Wetherbee, 1996) incluye un capítulo ("Proposal to expand the Reserva Natural Manejada of Loma Nalga de Maco, República Dominicana”) dedicado a esta área protegida. Otros colectores extranjeros que han visitado el área son Robert Woodruff (Florida State Collection of Arthropods), especialista en escarabajos y además colector de muchos otros insectos nocturnos y Thomas Henry (Systematic Entomology Lab, USDA), quien estudia principalmente la familia Miridae (Hemiptera).

Varios colectores dominicanos han muestreado los artrópodos en este parque nacional y sus alrededores. Las colectas entomológicas que se han llevado a cabo en el área, en su mayoría, han sido manuales con redes entomológicas y también utilizando luz ultravioleta (UV) para atraer insectos a una sábana blanca o a una trampa. Pero localmente no se han producido publicaciones en donde se registren insectos del área.

Las colecciones del Museo Nacional de Historia Natural de Santo Domingo (MNHNSD) y el Instituto de Investigaciones Botánicas y Zoológicas (IIBZ), de la Universidad Autónoma de Santo Domingo (UASD), contienen muchos especímenes colectados en el lugar, principalmente mariposas. Aún así, las especies identificadas son sólo una pequeña muestra de la entomofauna total del área. Es seguro que los variados ambientes representados en la Loma Nalga de Maco y alrededores contienen una proporción apreciable de los artrópodos que habitan la isla.

\section{RECOMENDACIONES}

Nunca se ha realizado ningún intento coordinado para evaluar las verdaderas dimensiones de la diversidad artropodológica en el Parque Nacional Loma Nalga de Maco. Recomendamos realizar un inventario que establezca parámetros básicos de la diversidad de especies. Dados los limitados recursos de tiempo y disponibilidad de expertos, se deberían seleccionar como prioritarios algunos grupos y hábitats significativos. Un problema particular de La Hispaniola es que la caracterización taxonómica de prácticamente todos los grupos de artrópodos es todavía muy preliminar. Para mejorar en este sentido, se necesitan más estudios taxonómicos y trabajos que resuman los conocimientos ya adquiridos en grupos particulares. 
Tabla 1. Resumen de la diversidad conocida de insectos en El Parque Nacional Loma Nalga de Maco y alrededores.

\begin{tabular}{|ll|}
\hline Orden & Especies \\
\hline Coleoptera & 12 \\
Diptera & 3 \\
Dictyoptera & 4 \\
Hemiptera & 18 \\
Lepidoptera & 48 \\
Odonata & 8 \\
Orthoptera & 12 \\
Phasmatodea & 1 \\
Trichoptera & 27 \\
\hline Total & 133 \\
\hline
\end{tabular}

Tabla 2. Lista de especies de insectos conocidos del Parque Nacional Loma Nalga de Maco y alrededores.

\begin{tabular}{|c|c|c|c|}
\hline Especies* & Referencia & Localidad & Notas \\
\hline \multicolumn{4}{|l|}{ COLEOPTERA } \\
\hline \multicolumn{4}{|l|}{ ELATERIDAE } \\
\hline $\begin{array}{l}\text { Ignelater dominicanensis } \\
\text { Fernández García \& Lozada Piña }\end{array}$ & $\begin{array}{l}\text { Fernández García \& } \\
\text { Lozada Piña, 2002:74 }\end{array}$ & Río Limpio & $\begin{array}{l}\text { Sólo conocida de Río Limpio. } \\
\text { Endémica de La Hispaniola }\end{array}$ \\
\hline \multicolumn{4}{|l|}{ LAMPYRIDAE } \\
\hline $\begin{array}{l}\text { Heterophotinus limpioensis } \\
\text { Kazantsev }\end{array}$ & Kazantsev, 2006:385 & Loma Nalga de Maco & $\begin{array}{l}\text { Sólo conocida de Loma Nalga } \\
\text { de Maco. Endémica de La } \\
\text { Hispaniola }\end{array}$ \\
\hline $\begin{array}{l}\text { Heterophotinus merielae } \\
\text { Kazantsev }\end{array}$ & Kazantsev, 2006:387 & Loma Nalga de Maco & $\begin{array}{l}\text { Sólo conocida de Loma Nalga } \\
\text { de Maco. Endémica de La } \\
\text { Hispaniola }\end{array}$ \\
\hline $\begin{array}{l}\text { Heterophotinus viridicolor } \\
\text { Kazantsev }\end{array}$ & Kazantsev, 2006:387 & Loma Nalga de Maco & $\begin{array}{l}\text { Sólo conocida de Loma Nalga } \\
\text { de Maco. Endémica de La } \\
\text { Hispaniola }\end{array}$ \\
\hline $\begin{array}{l}\text { Presbyolampis vegaensis } \\
\text { Kazantsev }\end{array}$ & Kazantsev, 2006:389 & Loma Nalga de Maco & Endémica de La Hispaniola \\
\hline \multicolumn{4}{|l|}{ SCARABAEIDAE } \\
\hline Neoathyreus biceps (Felsche) & Howden, 1996:1511 & Río Limpio & Endémica de La Hispaniola \\
\hline Phyllophaga imprima Sanderson & $\begin{array}{l}\text { Woodruff \& Sanderson, } \\
\text { 2004:81 }\end{array}$ & Río Limpio & Endémica de La Hispaniola \\
\hline $\begin{array}{l}\text { Phyllophaga jimenezi Woodruff \& } \\
\text { Sanderson }\end{array}$ & $\begin{array}{l}\text { Woodruff \& Sanderson, } \\
\text { 2004:87 }\end{array}$ & Río Limpio & Endémica de La Hispaniola \\
\hline Phyllophaga marcano Woodruff & $\begin{array}{l}\text { Woodruff \& Sanderson, } \\
\text { 2004:97 }\end{array}$ & 4 km E. Río Limpio & Endémica de La Hispaniola \\
\hline Phyllophaga mella Sanderson & $\begin{array}{l}\text { Woodruff \& Sanderson, } \\
\text { 2004:103 }\end{array}$ & Río Limpio & Endémica de La Hispaniola \\
\hline Phyllophaga recorta Sanderson & $\begin{array}{l}\text { Woodruff \& Sanderson, } \\
\text { 2004:130 }\end{array}$ & Río Limpio & Endémica de La Hispaniola \\
\hline Phyllophaga romana (Saylor) & $\begin{array}{l}\text { Woodruff \& Sanderson, } \\
\text { 2004:137 }\end{array}$ & Río Limpio & Endémica de La Hispaniola \\
\hline \multicolumn{4}{|l|}{ DIPTERA } \\
\hline ASILIDAE & & & \\
\hline
\end{tabular}

\footnotetext{
*Organizadas por Orden y Familia.
} 
Tabla 2 (continuación).

\begin{tabular}{|c|c|c|c|}
\hline Especies & Referencia & Localidad & Notas \\
\hline $\begin{array}{l}\text { Holcocephala indigena Scarbrough } \\
\text { \& Perez-Gelabert }\end{array}$ & $\begin{array}{l}\text { Scarbrough \& Perez- } \\
\text { Gelabert, 2006:84 }\end{array}$ & Río Limpio & Endémica de La Hispaniola \\
\hline \multicolumn{4}{|l|}{ CERATOPOGONIDAE } \\
\hline $\begin{array}{l}\text { Forcipomyia (Microhelea) } \\
\text { tettigonaris Wirth \& Castner }\end{array}$ & $\begin{array}{l}\text { Perez-Gelabert \& } \\
\text { Grogan, 1999:312 }\end{array}$ & Nalga de Maco & \\
\hline \multicolumn{4}{|l|}{ PHORIDAE } \\
\hline Dohrniphora divaricata (Aldrich) & $\begin{array}{l}\text { Kung \& Brown, } \\
\text { 2006:1936 }\end{array}$ & Río Limpio & \\
\hline \multicolumn{4}{|l|}{ HEMIPTERA } \\
\hline \multicolumn{4}{|l|}{ HETEROPTERA } \\
\hline \multicolumn{4}{|l|}{ RHYPAROCHOMIDAE } \\
\hline Neopamera bilobata (Say) & $\begin{array}{l}\text { Baranowski \& Slater, } \\
\text { 2005:152 }\end{array}$ & Río Limpio & \\
\hline Ozophora hispaniola Slater & $\begin{array}{l}\text { Baranowski \& Slater, } \\
\text { 2005:212 }\end{array}$ & Río Limpio & Endémica de La Hispaniola \\
\hline Ozophora parimpicta Baranowski & $\begin{array}{l}\text { Baranowski \& Slater, } \\
\text { 2005:225 }\end{array}$ & Río Limpio & \\
\hline Prytanes dissimilis (Barber) & $\begin{array}{l}\text { Baranowski \& Slater, } \\
\text { 2005:176 }\end{array}$ & Río Limpio & \\
\hline Prytanes formosa Barber & $\begin{array}{l}\text { Baranowski \& Slater, } \\
\text { 2005:177 }\end{array}$ & Río Limpio & \\
\hline \multicolumn{4}{|l|}{ GEOCORIDAE } \\
\hline $\begin{array}{l}\text { Pamphantus atrohumeralis } \\
\text { dominicanus Slater }\end{array}$ & $\begin{array}{l}\text { Baranowski \& Slater, } \\
\text { 2005:76 }\end{array}$ & Río Limpio & Endémica de La Hispaniola \\
\hline \multicolumn{4}{|l|}{ PENTATOMIDAE } \\
\hline $\begin{array}{l}\text { Acrosternum (Chinavia) insulani } \\
\text { Rolston }\end{array}$ & $\begin{array}{l}\text { Perez-Gelabert \& } \\
\text { Thomas, 2005:326 }\end{array}$ & Río Limpio & Endémica de La Hispaniola \\
\hline $\begin{array}{l}\text { Acrosternum (Chinavia) } \\
\text { marginatum (Palisot de Beauvois) }\end{array}$ & $\begin{array}{l}\text { Perez-Gelabert \& } \\
\text { Thomas, 2005:326 }\end{array}$ & Río Limpio & \\
\hline $\begin{array}{l}\text { Banasa punctatissima } \text { Barber \& } \\
\text { Bruner }\end{array}$ & $\begin{array}{l}\text { Perez-Gelabert \& } \\
\text { Thomas, 2005:329 }\end{array}$ & Río Limpio & \\
\hline Banasa zeteki Sailer & $\begin{array}{l}\text { Perez-Gelabert \& } \\
\text { Thomas, 2005:329 }\end{array}$ & Río Limpio & \\
\hline Brepholoxa rotundifrons Barber & $\begin{array}{l}\text { Perez-Gelabert \& } \\
\text { Thomas, 2005:344 }\end{array}$ & Río Limpio & \\
\hline Edessa bifida (Say) & $\begin{array}{l}\text { Perez-Gelabert \& } \\
\text { Thomas, 2005:345 }\end{array}$ & Loma de Las Tayotas & \\
\hline Fecelia nigridens (Walker) & $\begin{array}{l}\text { Perez-Gelabert \& } \\
\text { Thomas, 2005:334 }\end{array}$ & Río Limpio & \\
\hline Loxa viridis (Palisot de Beauvois) & $\begin{array}{l}\text { Perez-Gelabert \& } \\
\text { Thomas, 2005:335 }\end{array}$ & Loma de Las Tayotas & \\
\hline Oebalus ypsilongriseus (De Geer) & $\begin{array}{l}\text { Perez-Gelabert \& } \\
\text { Thomas, 2005:342 }\end{array}$ & Río Limpio & \\
\hline \multicolumn{4}{|l|}{ AUCHENORRHYNCHA } \\
\hline \multicolumn{4}{|l|}{ CICADIDAE } \\
\hline Chinaria vivianae Ramos & Ramos, 1983:63 & 4 km SE Río Limpio & Endémica de La Hispaniola \\
\hline Odopoea cariboea Uhler & $\begin{array}{l}\text { Identificada por Ruth H. } \\
\text { Bastardo }\end{array}$ & Río Limpio & $\begin{array}{l}\text { Endémica de La Hispaniola } \\
\text { Colección IIBZ }\end{array}$ \\
\hline \multicolumn{4}{|l|}{ MEMBRACIDAE } \\
\hline Ramosella dominicensis Ramos & $\begin{array}{l}\text { McKamey \& Dietz, } \\
\text { 1996:306 }\end{array}$ & Río Limpio & Endémica de La Hispaniola \\
\hline
\end{tabular}


Tabla 2 (continuación).

\begin{tabular}{|c|c|c|c|}
\hline Especies & Referencia & Localidad & Notas \\
\hline \multicolumn{4}{|l|}{ LEPIDOPTERA } \\
\hline \multicolumn{4}{|l|}{ ACROLOPHIDAE } \\
\hline Acrolophus arcasalis (Walker) & $\begin{array}{l}\text { Identificada por Donald } \\
\text { Davis }\end{array}$ & Río Limpio & Colección NMNH \\
\hline Acrolophus mimasalis (Walker) & $\begin{array}{l}\text { Identificada por Donald } \\
\text { Davis }\end{array}$ & Río Limpio & Colección NMNH \\
\hline \multicolumn{4}{|l|}{ TINEIDAE } \\
\hline Urodus mirella (Möschler) & Davis, 1986:90 & 4 km SE Río Limpio & \\
\hline \multicolumn{4}{|l|}{ HESPERIIDAE } \\
\hline Achlyodes mithridates sagra Evans & Schwartz, 1989:48 & 12 km NW Río Limpio & \\
\hline Chioides ixion (Plotz) & Schwartz, 1989:25 & 12 km NW Río Limpio & Endémica de La Hispaniola \\
\hline Choranthus haitensis Skinner & Schwartz, 1989:92 & 12 km NW Río Limpio & \\
\hline Erynnis zarucco Lucas & Schwartz, 1989:59 & 12 km NW Río Limpio & \\
\hline $\begin{array}{l}\text { Nyctelius nyctelius nyctelius } \\
\text { Latreille }\end{array}$ & Schwartz, 1989:112 & 16 km NW Río Limpio & \\
\hline Ochlodes batesi (Bell) & Schwartz, 1989:97 & $\begin{array}{l}\text { Cima Loma Nalga de } \\
\text { Maco }\end{array}$ & Endémica de La Hispaniola \\
\hline $\begin{array}{l}\text { Panoquina ocola distipuncta } \\
\text { Johnson \& Matusik }\end{array}$ & Schwartz, 1989:103 & 12 km NW Río Limpio & \\
\hline $\begin{array}{l}\text { Perichares philetes philetes } \\
\text { Gmelin }\end{array}$ & Schwartz, 1989:68 & 12 km NW Río Limpio & \\
\hline Polygonus leo leo (Gmelin) & Schwartz, 1989:23 & 12 km NW Río Limpio & \\
\hline $\begin{array}{l}\text { Proteides mercurius sanchesi Bell } \\
\& \text { Comstock }\end{array}$ & Schwartz, 1989:19 & 12 km NW Río Limpio & \\
\hline Urbanus proteus domingo Scudder & Schwartz, 1989:29 & 12 km NW Río Limpio & \\
\hline \multicolumn{4}{|l|}{ LYCAENIDAE } \\
\hline Leptotes cassius theonus Lucas & Schwartz, 1989:258 & 12 km NW Río Limpio & \\
\hline \multicolumn{4}{|l|}{ NYMPHALIDAE } \\
\hline Anetia jaegeri Ménétriés & Schwartz, 1989:480 & $\begin{array}{l}\text { Cima Loma Nalga de } \\
\text { Maco }\end{array}$ & \\
\hline $\begin{array}{l}\text { Archaeoprepona demophoon } \\
\text { insulicola (Godart) }\end{array}$ & Schwartz, 1989:376 & 12 km NW Río Limpio & \\
\hline Archimestra teleboas Ménétriés & Schwartz, 1989:336 & 16 km NW Río Limpio & Endémica de La Hispaniola \\
\hline $\begin{array}{l}\text { Calisto aleucosticha Correa \& } \\
\text { Schwartz }\end{array}$ & Schwartz, 1989:336 & $\begin{array}{l}\text { Los Guandules, cerca } \\
\text { de Guajayaco }\end{array}$ & Endémica de La Hispaniola \\
\hline Calisto confusa Lathy & Schwartz, 1989:160 & 12 km NW Río Limpio & Endémica de La Hispaniola \\
\hline Calisto galii Schwartz & $\begin{array}{l}\text { Schwartz \& Wetherbee } \\
\text { 1996:160 }\end{array}$ & Loma Nalga de Maco & Endémica de La Hispaniola \\
\hline Calisto obscura Michener & Schwartz, 1989:417 & $\begin{array}{l}16 \text { km NW Río Limpio; } \\
\text { Los Guandules }\end{array}$ & Endémica de La Hispaniola \\
\hline $\begin{array}{l}\text { Calisto wetherbeei Schwartz \& } \\
\text { González }\end{array}$ & $\begin{array}{l}\text { Schwartz \& González, } \\
\text { 1989:2 }\end{array}$ & Loma Nalga de Maco & $\begin{array}{l}\text { Endémica de La Hispaniola. } \\
\text { Sólo conocida de la cima de } \\
\text { Loma Nalga de Maco }\end{array}$ \\
\hline Colobura dirce wolcotti Comstock & Schwartz, 1989:354 & 12 km NW Río Limpio & \\
\hline Danaus cleophile Godart & Schwartz, 1989:467 & 12 km NW Río Limpio & \\
\hline Dryas iulia fucatus (Boddaert) & Schwartz, 1989:467 & 12 km NW Río Limpio & \\
\hline Eueides isabella melphis (Godart) & Schwartz, 1989:277 & 12 km NW Río Limpio & \\
\hline Euptoieta hegesia hegesia Cramer & Schwartz, 1989:289 & 16 km NW Río Limpio & \\
\hline
\end{tabular}


Tabla 2 (continuación).

\begin{tabular}{|c|c|c|c|}
\hline Especies & Referencia & Localidad & Notas \\
\hline Junonia genoveva (Cramer) & Schwartz, 1989:307 & 12 km NW Río Limpio & \\
\hline $\begin{array}{l}\text { Lucinia cadma torrebia } \\
\text { (Ménétriés) }\end{array}$ & Schwartz, 1989:340 & 16 km NW Río Limpio & \\
\hline Lycorea halia cleobaea (Godart) & Schwartz, 1989:471 & 12 km NW Río Limpio & \\
\hline $\begin{array}{l}\text { Marpesia eleuchea dospassosi } \\
\text { Munroe }\end{array}$ & Schwartz, 1989:360 & 12 km NW Río Limpio & \\
\hline \multicolumn{4}{|l|}{ PAPILIONIDAE } \\
\hline $\begin{array}{l}\text { Battus polydamas polycrates } \\
\text { Hopffer }\end{array}$ & Schwartz, 1989:119 & 12 km NW Río Limpio & \\
\hline Battus zetides Munroe & Schwartz, 1989:115 & $\begin{array}{l}\text { Cima Loma Nalga de } \\
\text { Maco }\end{array}$ & Endémica de La Hispaniola \\
\hline $\begin{array}{l}\text { Heraclides androgeus epidaurus } \\
\text { Godman \& Salvin }\end{array}$ & Schwartz, 1989:135 & 12 km NW Río Limpio & \\
\hline Heraclides machaonides Esper & Schwartz, 1989:131 & 12 km NW Río Limpio & Endémica de La Hispaniola \\
\hline Heraclides pelaus imerius (Godart) & Schwartz, 1989:142 & 12 km NW Río Limpio & \\
\hline \multicolumn{4}{|l|}{ PIERIDAE } \\
\hline Anteos clorinde (Godart) & Schwartz, 1989:200 & 16 km NW Río Limpio & \\
\hline Anteos maerula Fabricius & Schwartz, 1989:197 & 16 km NW Río Limpio & \\
\hline $\begin{array}{l}\text { Aphrissa statira hispaniolae } \\
\text { Munroe }\end{array}$ & Schwartz, 1989:225 & 12 km NW Río Limpio & \\
\hline Dismorphia spio Godart & Schwartz, 1989:226 & 12 km NW Río Limpio & \\
\hline Eurema daira palmira Poey & Schwartz, 1989:173 & 12 km NW Río Limpio & \\
\hline Eurema lisa eutherpe Ménétriés & Schwartz, 1989:158 & $\begin{array}{l}12 \text { \& } 16 \text { km NW Río } \\
\text { Limpio }\end{array}$ & \\
\hline Eurema nicippe Cramer & Schwartz, 1989:184 & 12 km NW Río Limpio & \\
\hline $\begin{array}{l}\text { Eurema proterpia proterpia } \\
\text { Fabricius }\end{array}$ & Schwartz, 1989:170 & 12 km NW Río Limpio & \\
\hline Phoebis agarithe antillia Brown & Schwartz, 1989:211 & 12 km NW Río Limpio & \\
\hline Phoebis argante rorata Butler & Schwartz, 1989:208 & 12 km NW Río Limpio & \\
\hline Phoebis sennae sennae Linnaeus & Schwartz, 1989:213 & 12 km NW Río Limpio & \\
\hline \multicolumn{4}{|l|}{ DICTYOPTERA } \\
\hline \multicolumn{4}{|l|}{ BLATTARIA } \\
\hline Epilampra sp. & $\begin{array}{l}\text { Identificada por D. E. } \\
\text { Perez-Gelabert }\end{array}$ & Río Limpio & Colección NMNH \\
\hline Neoblattella sp. & $\begin{array}{l}\text { Identificada por D. E. } \\
\text { Perez-Gelabert }\end{array}$ & Río Limpio & Colección NMNH \\
\hline \multicolumn{4}{|l|}{ MANTODEA } \\
\hline $\begin{array}{l}\text { Epaphrodita musarum (Palisot de } \\
\text { Beauvois) }\end{array}$ & $\begin{array}{l}\text { Lombardo \& Perez- } \\
\text { Gelabert, 2004:36 }\end{array}$ & Río Limpio & Endémica de La Hispaniola \\
\hline Gonatista phryganoides (Serville) & $\begin{array}{l}\text { Lombardo \& Perez- } \\
\text { Gelabert, 2004:36 }\end{array}$ & 4 km SE Río Limpio & Endémica de La Hispaniola \\
\hline \multicolumn{4}{|l|}{ ODONATA } \\
\hline \multicolumn{4}{|l|}{ ZYGOPTERA } \\
\hline \multicolumn{4}{|l|}{ COENAGRIONIDAE } \\
\hline Enallagma coecum (Hagen) & Flint et al., 2006: 69 & Río Limpio & \\
\hline Ischnura ramburii (Selys) & Flint et al., 2006: 70 & Río Limpio & \\
\hline
\end{tabular}


Tabla 2 (continuación).

\begin{tabular}{|c|c|c|c|}
\hline Especies & Referencia & Localidad & Notas \\
\hline \multicolumn{4}{|l|}{ SYNLESTIDAE } \\
\hline Phyllolestes ethelae Christiansen & Flint et al., 2006:73 & Río Limpio & Endémica de La Hispaniola \\
\hline \multicolumn{4}{|l|}{ ANISOPTERA } \\
\hline \multicolumn{4}{|l|}{ LIBELLULIDAE } \\
\hline Dythemis rufinervis (Burmeister) & Flint et al., 2006:75 & Río Limpio & \\
\hline Erythemis vesciculosa (Fabricius) & Flint et al., 2006:76 & Río Limpio & \\
\hline Macrothemis celeno (Selys) & Flint et al., 2006:78 & Río Limpio & \\
\hline Orthemis ferruginea (Burmeister) & Flint et al., 2006:79 & Río Limpio & \\
\hline Scapanea frontalis (Burmeister) & Flint et al., 2006:80 & Loma de Las Tayotas & \\
\hline \multicolumn{4}{|l|}{ ORTHOPTERA } \\
\hline \multicolumn{4}{|l|}{ TETRIGIDAE } \\
\hline Paratettix freygessneri I. Bolívar & $\begin{array}{l}\text { Identificada por D. E. } \\
\text { Perez-Gelabert }\end{array}$ & Río Limpio & Colección NMNH \\
\hline \multicolumn{4}{|l|}{ EPISACTIDAE } \\
\hline Antillacris eumenes Perez et al. & Perez et al., 1997:143 & $\begin{array}{l}\text { Loma Nalga de Maco } \\
(\sim 1200 \mathrm{~m})\end{array}$ & $\begin{array}{l}\text { Endémica de La Hispaniola. } \\
\text { Sólo conocida de Loma Nalga } \\
\text { de Maco }\end{array}$ \\
\hline \multicolumn{4}{|l|}{ ACRIDIDAE } \\
\hline $\begin{array}{l}\text { Dellia roseomaculata } \text { Perez- } \\
\text { Gelabert \& Otte }\end{array}$ & $\begin{array}{l}\text { Perez-Gelabert \& Otte, } \\
\text { 1999:5 }\end{array}$ & Loma de Las Tayotas & Endémica de La Hispaniola \\
\hline Hispanacris oreades Perez et al. & $\begin{array}{l}\text { Perez-Gelabert, 2000: } \\
338\end{array}$ & Loma de Las Tayotas & Endémica de La Hispaniola \\
\hline Orphulella punctata (De Geer) & $\begin{array}{l}\text { Identificada por D. E. } \\
\text { Perez-Gelabert }\end{array}$ & Río Limpio & Colección NMNH \\
\hline \multicolumn{4}{|l|}{ GRYLLIDAE } \\
\hline $\begin{array}{l}\text { Cycloptilum clandestinum Otte \& } \\
\text { Perez-Gelabert }\end{array}$ & $\begin{array}{l}\text { Otte \& Perez-Gelabert, } \\
\text { 2009:743 }\end{array}$ & Loma de Las Tayotas & Endémica de La Hispaniola \\
\hline \multicolumn{4}{|l|}{ TETTIGONIIDAE } \\
\hline Anaulocomera sp. & $\begin{array}{l}\text { Identificada por D. E. } \\
\text { Perez-Gelabert }\end{array}$ & Río Limpio & Colección NMNH \\
\hline Conocephalus sp. & $\begin{array}{l}\text { Identificada por D. E. } \\
\text { Perez-Gelabert }\end{array}$ & $\begin{array}{l}\text { Río Limpio; Loma de } \\
\text { Las Tayotas }\end{array}$ & Colección NMNH \\
\hline Erechthis sp. & $\begin{array}{l}\text { Identificada por D. E. } \\
\text { Perez-Gelabert }\end{array}$ & Río Limpio & Colección NMNH \\
\hline Neoconocephalus sp. & $\begin{array}{l}\text { Identificada por D. E. } \\
\text { Perez-Gelabert }\end{array}$ & Río Limpio & Colección NMNH \\
\hline $\begin{array}{l}\text { Polyancistrus serrulatus Palisot de } \\
\text { Beauvois }\end{array}$ & $\begin{array}{l}\text { Identificada por D. E. } \\
\text { Perez-Gelabert }\end{array}$ & $\begin{array}{l}\text { Loma Nalga de Maco; } \\
\text { Río Limpio }\end{array}$ & $\begin{array}{l}\text { Endémica de La Hispaniola } \\
\text { Colección NMNH }\end{array}$ \\
\hline Turpilia sp. & $\begin{array}{l}\text { Identificada por D. E. } \\
\text { Perez-Gelabert }\end{array}$ & Río Limpio & Colección NMNH \\
\hline \multicolumn{4}{|l|}{ PHASMATODEA } \\
\hline \multicolumn{4}{|l|}{ PSEUDOPHASMATIDAE } \\
\hline $\begin{array}{l}\text { Malacomorpha hispaniola Conle } \\
\text { et al. }\end{array}$ & Conle et al., 2008:22 & $\begin{array}{l}\text { Loma de Las Tayotas; } \\
\text { Río Limpio }\end{array}$ & Endémica de La Hispaniola \\
\hline \multicolumn{4}{|l|}{ TRICHOPTERA } \\
\hline \multicolumn{4}{|l|}{ CALAMOCERATIDAE } \\
\hline Phylloicus pulchrus Flint & Flint \& Sykora, 2004:3 & 4 km SE Río Limpio & \\
\hline
\end{tabular}


Tabla 2 (continuación).

\begin{tabular}{|c|c|c|c|}
\hline Especies & Referencia & Localidad & Notas \\
\hline \multicolumn{4}{|l|}{ ECNOMIDAE } \\
\hline Austrotinodes labiatus Flint & Flint \& Sykora, 2004:4 & 4 km SE Río Limpio & Endémica de La Hispaniola \\
\hline \multicolumn{4}{|l|}{ GLOSSOSOMATIDAE } \\
\hline Campsiophora pedophila Flint & Flint \& Sykora, 2004:5 & Río Limpio & \\
\hline Cariboptila caab Botosaneanu & Flint \& Sykora, 2004:5 & 4 km SE Río Limpio & \\
\hline Cariboptila hispaniolica Flint & Flint \& Sykora, 2004:6 & Río Limpio & \\
\hline \multicolumn{4}{|l|}{ HELICOPSYCHIDAE } \\
\hline $\begin{array}{l}\text { Helicopsyche (Feropsyche) } \\
\text { altercoma Botosaneanu \& Flint }\end{array}$ & Flint \& Sykora, 2004:8 & Río Limpio & Endémica de La Hispaniola \\
\hline $\begin{array}{l}\text { Helicopsyche (Feropsyche) } \\
\text { dominicana Botosaneanu \& Flint }\end{array}$ & Flint \& Sykora, 2004:8 & Río Limpio & Endémica de La Hispaniola \\
\hline $\begin{array}{l}\text { Helicopsyche (Feropsyche) } \\
\text { poliocheta Flint \& Sykora }\end{array}$ & Flint \& Sykora, 2004:11 & 4 km SE Río Limpio & \\
\hline \multicolumn{4}{|l|}{ HYDROBIOSIDAE } \\
\hline Atopsyche conventica Flint & Flint, 1974:4 & Río Limpio & Endémica de La Hispaniola \\
\hline Atopsyche davisorum Flint & Flint, 1974:4 & Río Limpio & Endémica de La Hispaniola \\
\hline \multicolumn{4}{|l|}{ CALOSOPSYCHIDAE } \\
\hline Calosopsyche domingensis (Banks) & Flint \& Sykora, 2004:19 & 4 km SE Río Limpio & Endémica de La Hispaniola \\
\hline $\begin{array}{l}\text { Smicridea (Smicridea) } \\
\text { brunnescens Flint \& Sykora }\end{array}$ & Flint \& Sykora, 2004:22 & Río Limpio & Endémica de La Hispaniola \\
\hline $\begin{array}{l}\text { Smicridea (Smicridea) comma } \\
\text { Banks }\end{array}$ & Flint \& Sykora, 2004:23 & Río Limpio & Endémica de La Hispaniola \\
\hline $\begin{array}{l}\text { Smicridea (Smicridea) duarte Flint } \\
\text { \& Sykora }\end{array}$ & Flint \& Sykora, 2004:24 & Río Limpio & Endémica de La Hispaniola \\
\hline \multicolumn{4}{|l|}{ HYDROPTILIDAE } \\
\hline Hydroptila ditalea Flint & Flint \& Sykora, 2004:31 & Río Limpio & \\
\hline $\begin{array}{l}\text { Hydroptila dominicana } \\
\text { Botosaneanu }\end{array}$ & Flint \& Sykora, 2004:31 & Río Limpio & Endémica de La Hispaniola \\
\hline Leucotricha tubifex Flint & Flint \& Sykora, 2004:32 & 4 km SE Río Limpio & \\
\hline Ochrotrichia seiba Flint \& Sykora & Flint \& Sykora, 2004:38 & Río Limpio & Endémica de La Hispaniola \\
\hline $\begin{array}{l}\text { Oxyethira (Mesotrichia) ortizorum } \\
\text { Botosaneanu }\end{array}$ & Flint \& Sykora, 2004:41 & Río Limpio & Endémica de La Hispaniola \\
\hline $\begin{array}{l}\text { Oxyethira (Loxotrichia) janella } \\
\text { Denning }\end{array}$ & Flint \& Sykora, 2004:43 & Río Limpio & \\
\hline $\begin{array}{l}\text { Oxyethira (Loxotrichia) } \\
\text { puertoricensis Flint }\end{array}$ & Flint \& Sykora, 2004:44 & Río Limpio & \\
\hline $\begin{array}{l}\text { Oxyethira (Dampfitrichia) tega } \\
\text { Flint }\end{array}$ & Flint \& Sykora, 2004:45 & Río Limpio & \\
\hline \multicolumn{4}{|l|}{ PHILOPOTAMIDAE } \\
\hline $\begin{array}{l}\text { Chimarra (Curgia) gilvimacula } \\
\text { Flint }\end{array}$ & Flint \& Sykora, 2004:50 & Río Limpio & Endémica de La Hispaniola \\
\hline $\begin{array}{l}\text { Chimarra (Otarrha) spinulifera } \\
\text { galalcha Botosaneanu }\end{array}$ & Flint \& Sykora, 2004:52 & Río Limpio & Endémica de La Hispaniola \\
\hline POLYCENTROPODIDAE & & & \\
\hline
\end{tabular}


Tabla 2 (continuación).

\begin{tabular}{|l|c|c|c|}
\hline \multicolumn{1}{|c|}{ Especies } & Referencia & Localidad & Notas \\
\hline Antillopsyche demma Botosaneanu & Flint \& Sykora, 2004:52 & Río Limpio & Endémica de La Hispaniola \\
Polycentropus jeldesi Flint & Flint \& Sykora, 2004:54 & Río Limpio & Endémica de La Hispaniola \\
Polycentropus vanderpooli Flint & Flint \& Sykora, 2004:54 & Río Limpio & Endémica de La Hispaniola \\
\hline
\end{tabular}

\section{AGRADECIMIENTOS}

A Donald y Mignon Davis (U.S. National Museum of Natural History, Smithsonian Institution) por datos y comentarios sobre su visita a Río Limpio en 1973.

\section{LITERATURA CITADA}

Baranowski, R. M. y J. A. Slater. 2005. The Lygaeidae of the West Indies. University of Florida, IFAS, Florida Agricultural Experiment Station, Bulletin 402, 266 pp.

Conle, O. V., F. H. Hennemann y D. E. Perez-Gelabert. 2008. Studies on Neotropical Phasmatodea II: revision of the genus Malacomorpha Rehn, 1906, with the description of seven new species (Phasmatodea: Pseudophasmatidae: Pseudophasmatini). Zootaxa, 1748: 1-64.

Davis, D. R. 1986. Neotropical Tineidae, I: The types of H. B. Möschler(Lepidoptera: Tineoidea). Proceedings of the Entomological Society of Washington, 88: 83-92.

Fernández García, I. y A. Lozada Piña. 2002. Descripción de una especie nueva de Ignelater (Coleoptera: Elateridae: Pyrophorinae) de República Dominicana. Avicennia, 15: 73-76.

Flint, O. S., Jr. 1974. Studies on Neotropical caddisflies 18: new species of Rhyacophylidae and Glossosomatidae (Trichoptera). Smithsonian Contributions to Zoology, 169: 1-30.

Flint, O. S., Jr. y J. L. Sykora. 2004. Caddisflies of Hispaniola, with special reference to the Dominican Republic (Insecta: Trichoptera). Annals of the Carnegie Museum, 73: 1-60.

Flint, O. S., Jr., R. H. Bastardo y D. E. Perez-Gelabert. 2006. Distribution of the Odonata of the Dominican Republic. Bulletin of American Odonatology, 9: 67-84.

Howden, H. F. 1996. West Indian Neoathyreus and their over-water dispersal (Coleoptera: Scarabaeidae: Geotrupinae). Journal of Natural History, 30: 1503-1515.

Kazantsev, S. V. 2006. New firefly taxa from Hispaniola and Puerto Rico (Coleoptera: Lampyridae), with notes on biogeography. Russian Entomological Journal, 15: 367-392.

Kung, G. A. y B. V. Brown. 2006. Review of the Caribbean species of Dohrniphora Dahl (Diptera: Phoridae). Journal of Natural History, 40: 1931-1945.

Lombardo, F. y D. E. Perez-Gelabert. 2004. The mantids of Hispaniola (Mantodea), with the description of two new species. Boletín de la Sociedad Entomológica Aragonesa, 34: 35-48.

McKamey, S. H. y L. L. Dietz. 1996. Generic revision of the New World tribe Hoplophorionini (Hemiptera: Membracidae: Membracinae). Systematic Entomology, 21: 295-342. 
Otte, D. y D. E. Perez-Gelabert. 2009. Caribbean Crickets. The Orthopterists' Society, Philadelphia, 792 pp.

Perez-Gelabert, D. E. 2000. New locality and island records for seventeen species of West Indian grasshoppers (Orthoptera: Caelifera). Caribbean Journal of Science, 36: 335-340.

Perez-Gelabert, D. E. 2008. Arthropods of Hispaniola (Dominican Republic and Haiti): a checklist and bibliography. Zootaxa, 1831, 1-530.

Perez-Gelabert, D. E. y D. Otte. 1999. Dos nuevas especies de saltamontes del género Dellia (Orthoptera: Acrididae) de la República Dominicana. Novitates Caribaea, 1: 1-13.

Perez-Gelabert, D. E. y D. B. Thomas. 2005. Stink bugs (Heteroptera: Pentatomidae) of the island of Hispaniola, with the description of seven new species from the Dominican Republic. Boletín de la Sociedad Entomológica Aragonesa, 37: 319-352.

Perez, D. E., G. O. Dominici, B. Hierro y D. Otte. 1995. New grasshopper genera and species from the Dominican Republic (Hispaniola) (Acridoidea: Acrididae). Transactions of the American Entomological Society, 121: 153-171.

Perez, D. E., G. O. Dominici, B. Hierro y D. Otte. 1997. New eumastacid grasshopper taxa (Orthoptera: Eumastacidae: Episactinae) from Hispaniola, including a fossil new genus and species from the Dominican amber. Journal of Orthoptera Research, 6: 139-151.

Perez-Gelabert, D. E. y W. L. Grogan, Jr. 1999. Forcipomyia (Microhelea) tettigonaris (Diptera: Ceratopogonidae) parasitizing katydids (Orthoptera: Tettigoniidae) in the Dominican Republic. Entomological News, 110: 311-314.

Ramos, J.A. 1983. Sinópsis de las cigarras de la República Dominicana (Cicadoidea, HomopteraAuchenorhyncha). Caribbean Journal of Science, 19: 61-68.

Scarbrough, A. S. y D. E. Perez-Gelabert. 2006. A review of the asilid (Diptera) fauna from Hispaniola, with six genera new to the island, fifteen new species and checklist. Zootaxa, 1381: 1-91.

Schwartz, A. 1989. The Butterflies of Hispaniola. University of Florida Press, Gainesville, $580 \mathrm{pp}$.

Schwartz, A. y D. K. Wetherbee. 1996. Calisto (Lepidoptera: Satyridae) of the Hispaniolan western Cordillera Central. Caribbean Journal of Science, 32: 158-165.

Schwartz, A. y F. Gonzalez. 1989. A new species of Calisto from Hispaniola. Bulletin of the Allyn Museum, 117: 1-5.

Wetherbee, D. K. 1996. Proposal to expand the Reserva Natural Manejada of Loma Nalga Maco, República Dominicana. Pp. 310-345. En: La Xaiba prieta and La Xaiba Piñita (Epilobocera: Decapoda) in Hispaniola, and 20+ further contributions on Hispaniolan fauna. Printed by the author, Santo Domingo, 465 pp.

Woodruff, R. E. y M. W. Sanderson. 2004. Revision of the Phyllophaga of Hispaniola (Coleoptera: Scarabaeidae: Melolonthinae). Insecta Mundi, 18: 1-154. 